\title{
Stable acceptor-donor-acceptor type open-shell diradical with ultra- narrow bandgap and efficient photothermal conversion
}

\author{
Weixuan Liang, Zejun Wang, Jiaxing Huang, Xinyang Tao, Yuan Li* \\ Open-shell diradical, donor-acceptor, near-infrared absorption, narrow bandgap, photothermal conversion
}

\begin{abstract}
It has been reported recently that open-shell diradical character could exist in narrow bandgap donor-acceptor (D-A) organic semiconductors in our previous work. The A-D-A type molecules play as important role in the organic electronic such as donors and acceptors (ITIC and Y6) in organic solar cells. However, their relatively poor chemical and photostability prevent their industrial application. In this work, we reported a stable A-D-A type open-shell diradical, named LY1-4Cl, by replacing the 2-(3-oxo-2,3-dihydro-1H-inden-1-yli-dene)malononitrile (IC)-series end group of typical closed-shell nonfullerene acceptor IT-4Cl. The new electron-withdrawing building block could give LY1-4Cl the redshift near-infrared (NIR) absorption, the smaller bandgap and highly enhanced photostability comparing with IT-4Cl. The open-shell diradical character of LY1-4Cl originates from the formation of quinoid diradical form. Moreover, benefiting from the broad NIR absorption and radical-promoted nonradiative transition, LY1-4Cl displayed high photothermal properties that an increasing temperature of $188^{\circ} \mathrm{C}$ was recorded within $60 \mathrm{~s}$ under $808 \mathrm{~nm}$ laser irradiation of $0.8 \mathrm{~W} \mathrm{~cm}^{-2}$. This research provides a new strategy to design IC-free A-D-A type open-shell quinoid diradical with ultra-narrow bandgap, good photostability and efficient photothermal conversion.
\end{abstract}

Organic radicals are of great interest due to their one or more unpaired electrons leading to unique physicochemical properties and potential applications in organic/perovskite solar cells, ${ }^{1-4}$ organic field-effect transistors ${ }^{5,6}$ as well as organic photothermal materials. ${ }^{7-10}$ One of the most famous radical systems is open-shell diradicals based on Chichibabin's hydrocarbon derivatives, whose driving force is the recovery of aromaticity (Ar) of the quinoidal $(\mathrm{Qu})$ rings, then producing two unpaired electrons (Figure 1A). ${ }^{11-13}$ Several strategies including steric protection, electron delocalization, introduction of electronwithdrawing groups and heteroatom assistance have been applied to stabilize the highly reactive diradicals. ${ }^{14}$ Recent years have witnessed great progress of these types of diradicals, ${ }^{15-22}$ but it remains a challenging subject to further develop stable diradicals because of their complex synthetic routes.

With different formation and stabilization mechanisms of diradicals, another emerging radical system is donoracceptor (D-A) narrow bandgap organic semiconductors. ${ }^{12}$ Many compounds have been reported to exhibit obvious diradical character using strong electron-rich donors to combine with acceptor units such as benzo[1,2-c:4,5c']bis[1,2,5]thiadiazole ${ }^{23-27} \quad$ (BBT) and diketopyrrolopyrrole ${ }^{28-30}$ (DPP). Significantly, our group revealed that it is the open-shell quinoid resonance structure of D-A-D type narrow bandgap small molecules that results in their diradical character (Figure 1B), ${ }^{28,29}$ and further proposed the mechanism of aggregate-induced radical. ${ }^{30}$ Strong electron D-A planar structure is beneficial to achieve high intramolecular charge transfer (ICT) effect and to reduce the bandgap, contributing to the formation of quinoidal biradical resonance structure. ${ }^{29}$ However, there are few reports about A-D-A type organic small molecules that possess open-shell diradical character.

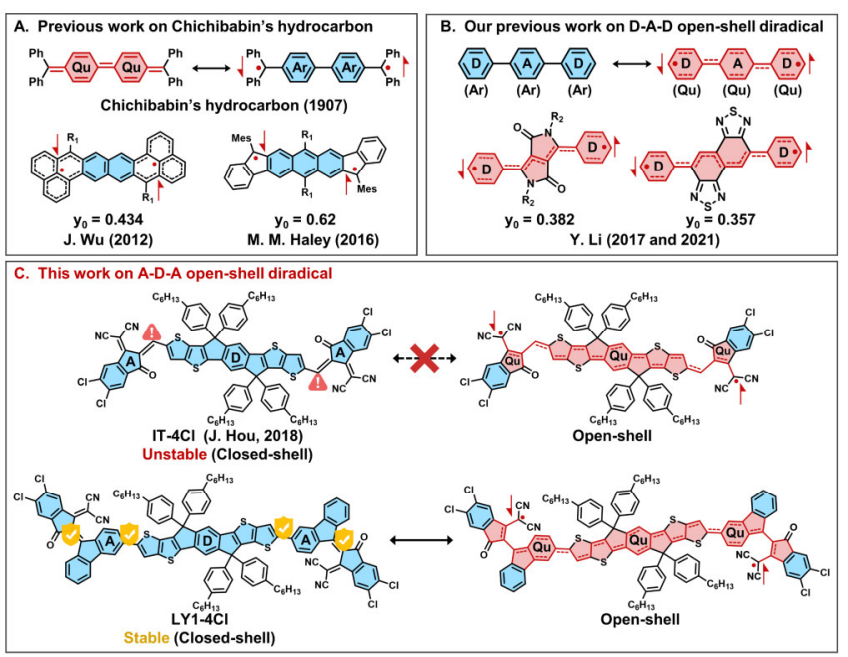

Figure 1. (A) Resonant structure of Chichibabin's hydrocarbon and classical examples of open-shell diradical driven by the recovery of aromaticity (Ar). (B) Open-shell D-A-D quinoid (Qu) diradical in our previous work. (C) The A-D-A type closed-shell acceptor IT$4 \mathrm{Cl}$ and open-shell diradical LY1-4Cl in this work.

Nowadays, A-D-A strategy has been successfully applied in the design of non-fullerene acceptors (NFAs) with nearinfrared (NIR) absorption and narrow bandgap. ${ }^{31-34}$ The 2(3-oxo-2,3-dihydro-1H-inden-1-yli-dene)malononitrile (IC)-series are the most popular electron-withdrawing groups in the synthesis of NFAs, but these NFAs containing vulnerable exocyclic double bonds could easily suffer from poor photostability. ${ }^{35-38}$ In addition, high performance A-D- 

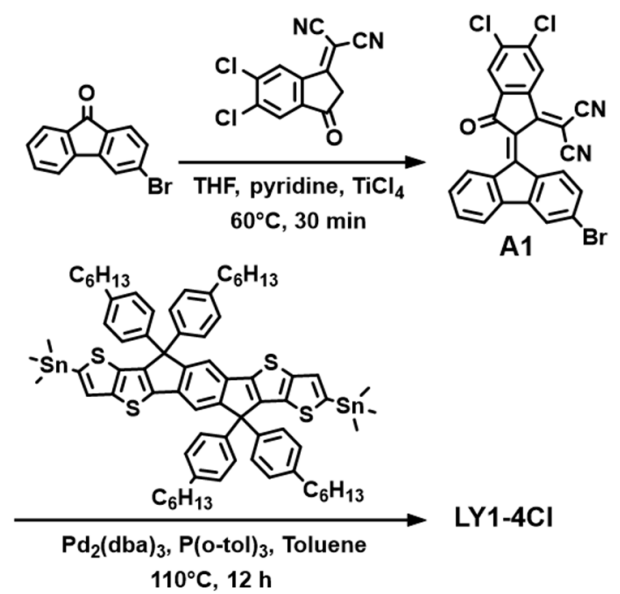

Scheme 1. Synthetic route of LY1-4Cl.

A type NFAs like ITIC series show closed-shell electronic structure and extremely weak electron spin resonance signal. ${ }^{29}$ Further reducing the bandgap is conducive to induce forming open-shell quinoidal structure and enhance non-radiative deactivation to attain efficient photothermal conversion. 9, 28, 29, 39-42 Thus, it is necessary to develop new acceptor units that not only could endow materials with improved stability but also have stronger electronwithdrawing ability to narrow the bandgap.

Herein, an IC-free ultra-narrow bandgap A-D-A type small molecule, namely LY1-4Cl, was well designed and simply synthesized by changing the electron-withdrawing unit of IT-4Cl reported by Hou's group ${ }^{43}$ (Figure 1C). Owing to the improved ICT effect, LY1-4Cl showed a significantly redshifted absorption even with insufficient planarity relative to IT-4Cl. In addition, the narrow bandgap and high HOMO level of LY1-4Cl lead to the formation of open-shell quinoid diradical character. The photostability of LY1-4Cl has greatly enhanced with its intrinsic structure stability, proving the effectiveness of our molecular design strategy. Moreover, the temperature of $\mathrm{LY} 1-4 \mathrm{Cl}$ powder could rapidly increase to $188^{\circ} \mathrm{C}$ under $808 \mathrm{~nm}$ laser irradiation $\left(0.8 \mathrm{~W} \mathrm{~cm}^{-2}\right)$, which is about $30 \%$ higher than that of IT- $4 \mathrm{Cl}$. Therefore, this study provides a facile synthesis of A-D-A type open-shell diradical to achieve broad NIR absorption and efficient photothermal conversion.

As shown in Scheme 1, the synthesis of LY1-4Cl contains a two-step facile reaction. The intermediate A1 was obtained from the Knoevenagel condensation between 3bromo-9H-fluoren-9-one and 2-(5,6-dichloro-3-oxo-2,3dihydro-1H-inden-1-ylidene)malononitrile using pyridine and $\mathrm{TiCl}_{4}$ as catalysts, in a good yield of $86 \%$. The desired compound LY1-4Cl was synthesized via Stille coupling reaction employing commercial indacenodithieno[3,2b]thiophene tin reagent (IDTT-Sn) to react with A1. The structure of target product was well characterized by Nuclear magnetic resonance (NMR) spectra and the highresolution matrix-assisted laser desorption/ionization time of flight (MALDI-TOF) in the supporting information (Figure S1-S3).

The UV-Vis absorption spectra of LY1-4Cl and IT-4Cl in chloroform solutions and thin films are presented in Figure 2A. The LY1-4Cl in chloroform solution displayed a maximum absorption peak at $811 \mathrm{~nm}$ with an obvious redshift of about $108 \mathrm{~nm}$ when compared with that of IT-4Cl
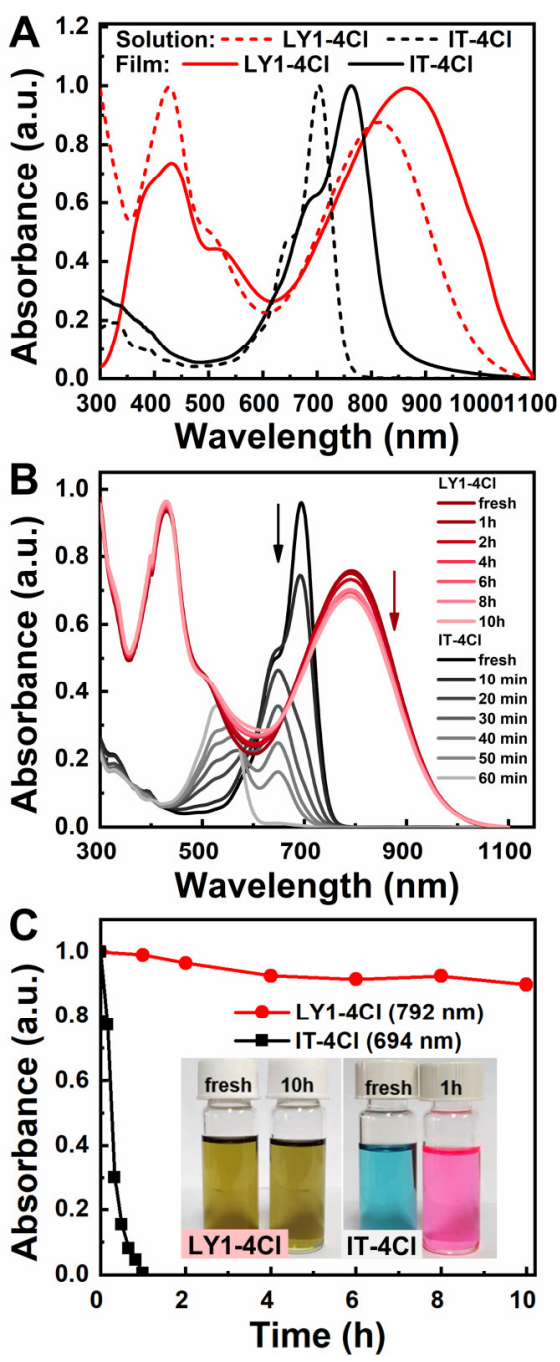

Figure 2. (A) UV-Vis absorption spectra of LY1-4Cl and IT-4Cl in chloroform solutions and thin films prepared from chloroform solution. (B) UV-vis absorption decay spectra of LY1-4Cl and IT-4Cl in chlorobenzene solution under the irradiation of $100 \mathrm{~W}$ tungsten lamp. (C) The decrease of absorption intensity and the inserted photo images of LY1-4Cl and IY-4Cl with increasing irradiation time.

solution. The LY1-4Cl film had a broad and strong absorption in the $600-1100 \mathrm{~nm}$ range, which can cover more sunshine radiation spectra than IT-4Cl. The absorption onset of LY1-4Cl and IT-4Cl films were $1100 \mathrm{~nm}$ and $825 \mathrm{~nm}$, respectively, with corresponding optical bandgaps of 1.13 and $1.50 \mathrm{eV}$, respectively. However, the maximum extinction coefficient of LY1-4Cl film was calculated to be $5.29 \times 10^{4} \mathrm{~cm}^{-1}$, which is lower than $1.26 \times 10^{5} \mathrm{~cm}^{-1}$ of IT- $4 \mathrm{Cl}$ film. ${ }^{44}$ These results are caused by the stronger electron withdrawing ability of the new acceptor unit, the extended $\pi$-conjugation, and the enhanced intramolecular charge transfer interaction of LY1-4Cl.45-47

To evaluate the photostability of LY1-4Cl and IT-4Cl in chlorobenzene solution, their absorption decay spectra with different irradiation times were recorded. The results were displayed in Figure 2B-2C. When exposed to one- 

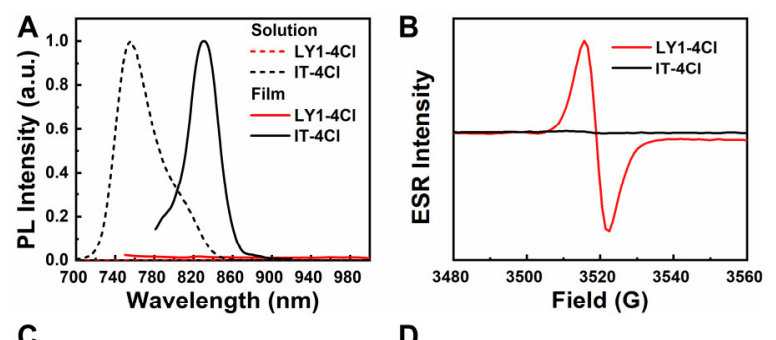

C

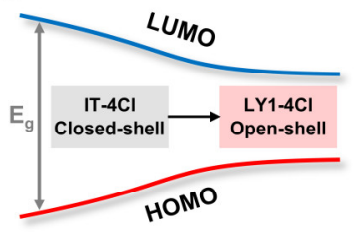

D

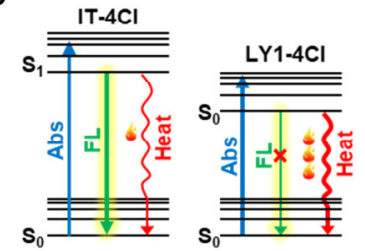

Figure 3. (A) Photoluminescence spectra of LY1-4Cl and IT-4Cl in chloroform solutions (excited at $650 \mathrm{~nm}$ ) and thin films (excited at $720 \mathrm{~nm}$ ). (B) ESR spectra of LY1-4Cl and IT-4Cl powders at the same mole amount (0.01 mmol). (C) Mechanism of the formation of open-shell structure. (D) Jablonski diagram illustrates the different energy loss pathways of photoexcited LY1-4Cl and IT-4Cl.

hour illumination, the absorption intensity of IT-4Cl at maximum absorption peak (694 $\mathrm{nm}$ ) reduced dramatically to zero along with the solution color changing from initial blue to pink, which suggests its entire photodegradation. Encouragingly, LY1-4Cl retained the unchanged solution color and about $90 \%$ of its original absorption intensity even after irradiation of ten hours, confirming enhanced photostability than IT-4Cl.

The energy levels of two materials were measured by cyclic voltammetry (CV) method (Figure S4). The energy level diagram of LY1-4Cl and IT-4Cl was shown in Figure S5 and the corresponding parameters were collected in Table S1. The HOMO/LUMO energy levels of LY1-4Cl and IT-4Cl were determined to be $-5.43 /-4.33$ and $-5.75 /-4.14$ $\mathrm{eV}$, respectively. Without changing the donor moiety IDTT, the LUMO energy level of LY1-4Cl was downshifted by 0.19 $\mathrm{eV}$ relative to IT-4Cl, consistent with its stronger electron-

withdrawing capability acceptor moiety. Meanwhile, it is worth noting that the HOMO energy level of LY1-4Cl was markedly increased by $0.32 \mathrm{eV}$, which resulted in the ultranarrow electrochemical bandgap of $1.10 \mathrm{eV}$, consistent with the optical bandgap.

To study the electron transition process in its excited state, we tested the photoluminescence (PL) spectrum of LY1-4Cl and IT-4Cl in chloroform solution and thin film. As shown in Figure 3A, the IT-4Cl in chloroform solution and film exhibited a strong fluorescence emission peak at 756 and $830 \mathrm{~nm}$, respectively, while on the contrary, almost complete fluorescence quenching was discovered in that of LY1-4Cl. This means that LY1-4Cl is a potential candidate for photothermal material because its excited-state energy is mainly dissipated through nonradiative transition, leading to efficient heat production.

The electron spinning resonance (ESR) spectroscopy was employed to characterize the radical properties of their powders (Figure 3B and Figure S6). IT-4Cl exhibited a very weak ESR signal owing to its closed-shell electronic structure. In contrast, a strong ESR signal with a g-factor of 2.0025 was recorded for $\mathrm{LY} 1-4 \mathrm{Cl}$. The result demonstrates the existence of open-shell electronic structure and carbon radical in $\mathrm{LY}-4 \mathrm{Cl}$, which can be attributed to its higher HOMO energy level and lower bandgap (Figure 3C). 28-30, 48

Considering the extended near-infrared absorption and radical-promoted nonradiative decay, LY1-4Cl may possess

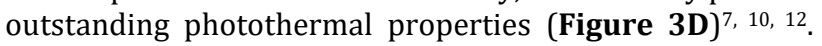
Thus, we used an infrared camera to monitor the temperature change of their powders $(10 \mathrm{mg}$ ) under 808 $\mathrm{nm}$ laser irradiation, to evaluate the photothermal performances of LY1-4Cl and IT-4Cl (Figure 4A and Figure S7). When exposed to $808 \mathrm{~nm}$ laser of $0.8 \mathrm{~W} \mathrm{~cm} \mathrm{~cm}^{-2}$ illumination density for $60 \mathrm{~s}$, the temperature of LY1-4Cl powder can rise rapidly to around $188{ }^{\circ} \mathrm{C}$ that is much higher than that $\left(148{ }^{\circ} \mathrm{C}\right)$ of $\mathrm{IT}-4 \mathrm{Cl}$ (Figure 4B). Furthermore, little difference of maximum temperature was observed for LY1-4Cl after ten cycles of heating and cooling, which illustrates its good photobleaching resistance and photothermal stability, while unexpectedly good stability was also found in IT-4Cl in the photothermal cyclic test (Figure 4C). It shows clearly that the maximum
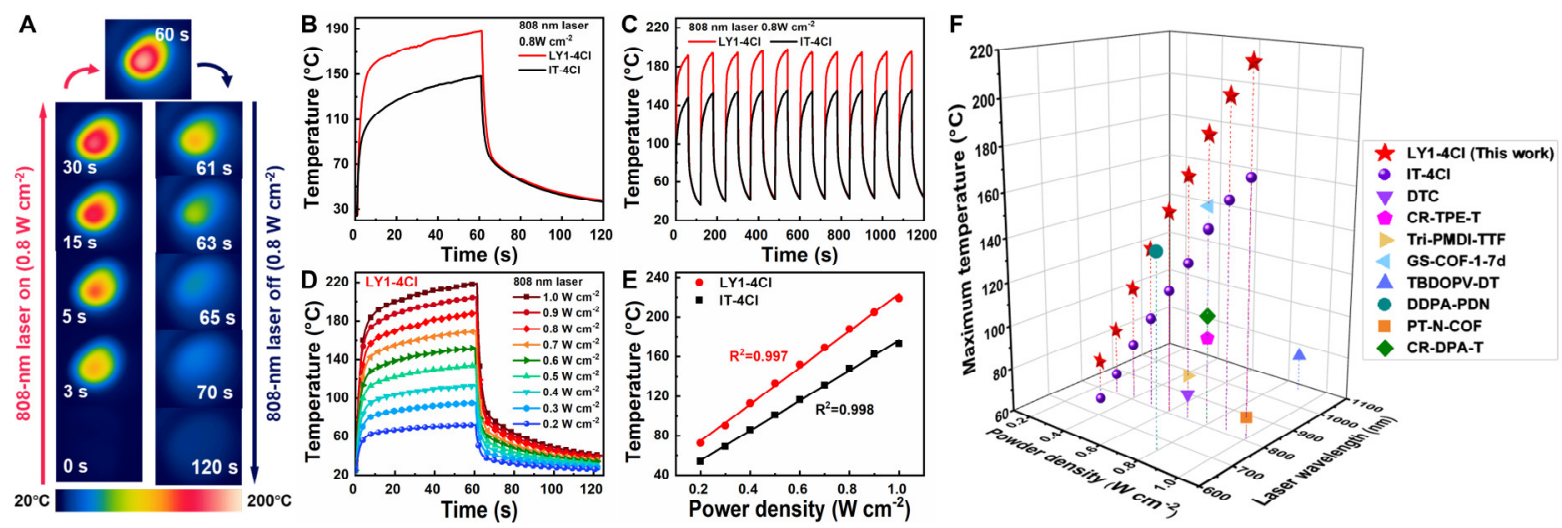

Figure 4. (A) Infrared thermal images of LY1-4Cl powder, (B) Photothermal conversion behavior of LY1-4Cl and IT-4Cl powders under $808 \mathrm{~nm}$ laser irradiation $\left(0.8 \mathrm{~W} \mathrm{~cm}^{-2}\right)$ and then turned off. (C) Photobleaching resistance property of LY1-4Cl and IT-4Cl powders during ten cycles of heating-cooling processes. (D) Photothermal conversion behavior of LY1-4Cl powder under $808 \mathrm{~nm}$ laser irradiation at different laser power $\left(0.2-1.0 \mathrm{~W} \mathrm{~cm}^{-2}\right)$. (E) Fitting lines of maximum temperature of LY1-4Cl and IT-4Cl in different power density. (F) Maximum temperature of reported photothermal materials under laser irradiation. 
temperature was the linear relationship with laser power densities for both LY1-4Cl and IT-4Cl (Figure 4D-4E and Figure S8). As shown in Figure 4F, these results demonstrate the superior photothermal properties compared with other reported organic photothermal materials (Table S2). 7, 9, 39, 49-55

In conclusion, an IC-free ultra-narrow bandgap openshell diradical named LY1-4Cl featuring an A-D-A structure was attained by a facile synthesis. Without changing the donor unit, LY1-4Cl had significant red-shift of absorption even with insufficient planarity in comparison with IT-4Cl, indicating the stronger electron-withdrawing ability of our acceptor unit. The higher HOMO energy level together with the smaller bandgap of $\mathrm{LY} 1-4 \mathrm{Cl}$ are conducive to the formation of open-shell quinoid diradical. More experiments discovered that both the photostability and photothermal properties of LY1-4Cl were higher than IT4Cl. These advantages above make LY1-4Cl possess potential application in solar-driven water evaporation and photothermal therapy. In the future, we expect to further extend the NIR absorption by improving the planarity between donor unit and acceptor unit based on this strategy. Overall, this work makes an important step toward nearinfrared A-D-A type material with high photostability and brings new insight in developing open-shell diradical with efficient photothermal conversion.

\section{ASSOCIATED CONTENT}

Supporting Information. This material is available free of charge.

\section{AUTHOR INFORMATION}

\section{Corresponding Author}

* Yuan Li - Institute of Polymer Optoelectronic Materials and Devices, State Key Laboratory of Luminescent Materials and Devices, South China University of Technology, Guangzhou, 510640, P. R. China. Email: celiy@scut.edu.cn

\section{Authors}

Weixuan Liang - Institute of Polymer Optoelectronic Materials and Devices, State Key Laboratory of Luminescent Materials and Devices, South China University of Technology, Guangzhou, 510640, P. R. China.

Zejun Wang - Institute of Polymer Optoelectronic Materials and Devices, State Key Laboratory of Luminescent Materials and Devices, South China University of Technology, Guangzhou, 510640, P. R. China.

Jiaxing Huang - Institute of Polymer Optoelectronic Materials and Devices, State Key Laboratory of Luminescent Materials and Devices, South China University of Technology, Guangzhou, 510640, P. R. China.

Xinyang Tao - Institute of Polymer Optoelectronic Materials and Devices, State Key Laboratory of Luminescent Materials and Devices, South China University of Technology, Guangzhou, 510640, P. R. China.

\section{Notes}

The authors declare no competing financial interest.

\section{ACKNOWLEDGMENT}

The work was financially supported by the Natural Science Foundation of China (51973063), the Tip-top Scientific and
Technical Innovative Youth Talents of Guangdong Special Support Program (2019TQ05C890), the Ministry of Science and Technology of the People's Republic of China (2017YFA0206600), the Pearl River S\&T Nova Program of Guangzhou (201710010194) and the Fund of Guangdong Provincial Key Laboratory of Luminescence from Molecular Aggregates (2019B030301003).

\section{REFERENCES}

(1) Ma, R.; Zeng, M.; Li, Y.; Liu, T.; Luo, Z.; Xu, Y.; Li, P.; Zheng, N.; Li, J.; Li, Y.; et al. Rational Anode Engineering Enables Progresses for Different Types of Organic Solar Cells. Adv. Energy Mater. 2021, 11 (23), 2100492.

(2) Zeng, M.; Wang, X.; Ma, R.; Zhu, W.; Li, Y.; Chen, Z.; Zhou, J.; Li, W.; Liu, T.; He, Z.; et al. Dopamine Semiquinone Radical Doped PEDOT:PSS: Enhanced Conductivity, Work Function and Performance in Organic Solar Cells. Adv. Energy Mater. 2020, 10 (25), 2000743.

(3) Xue, Q.; Liu, M.; Li, Z.; Yan, L.; Hu, Z.; Zhou, J.; Li, W.; Jiang, X.-F.; Xu, B.; Huang, F.; et al. Efficient and Stable Perovskite Solar Cells via Dual Functionalization of Dopamine Semiquinone Radical with Improved Trap Passivation Capabilities. Adv. Funct. Mater. 2018, 28 (18), 1707444.

(4) Zhang, Y.-H.; Li, Y. Interface materials for perovskite solar cells. Rare Metals 2021, 40 (11), 2993-3018.

(5) Yang, K.; Zhang, X.; Harbuzaru, A.; Wang, L.; Wang, Y.; Koh, C.; Guo, H.; Shi, Y.; Chen, J.; Sun, H.; et al. Stable Organic Diradicals Based on Fused Quinoidal Oligothiophene Imides with High Electrical Conductivity. J. Am. Chem. Soc. 2020, 142 (9), 4329-4340.

(6) Hu, X.; Wang, W.; Wang, D.; Zheng, Y. The electronic applications of stable diradicaloids: present and future. J. Mater. Chem. C 2018, 6 (42), 11232-11242.

(7) Chen, G.; Sun, J.; Peng, Q.; Sun, Q.; Wang, G.; Cai, Y.; Gu, X.; Shuai, Z.; Tang, B. Z. Biradical-Featured Stable Organic-SmallMolecule Photothermal Materials for Highly Efficient SolarDriven Water Evaporation. Adv. Mater. 2020,32 (29), 1908537. (8) Li, X.; Zhang, D.; Yin, C.; Lu, G.; Wan, Y.; Huang, Z.; Tan, J.; Li, S.; Luo, J.; Lee, C. S. A Diradicaloid Small Molecular Nanotheranostic with Strong Near-Infrared Absorbance for Effective Cancer Photoacoustic Imaging and Photothermal Therapy. ACS Appl. Mater. Interfaces 2021, 13 (14), 15983-15991. (9) Sun, J.; Zhao, E.; Liang, J.; Li, H.; Zhao, S.; Wang, G.; Gu, X.; Tang, B. Z. Diradical-Featured Organic Small-Molecule Photothermal Material with High-Spin State in Dimers for UltraBroadband Solar Energy Harvesting. Adv. Mater. 2022, 2108048. (10) Wang, Z.; Zhou, J.; Zhang, Y.; Zhu, W.; Li, Y. Accessing Highly Efficient Photothermal Conversion with Stable Open-Shell Aromatic Nitric Acid Radicals. Angew. Chem. Int. Ed. 2022, e202113653.

(11) Huang, Y.; Egap, E. Open-shell organic semiconductors: an emerging class of materials with novel properties. Polym $J$ 2018, $50(8), 603-614$.

(12) Chen, Z. X.; Li, Y.; Huang, F. Persistent and Stable Organic Radicals: Design, Synthesis, and Applications. Chem 2021, 7 (2), 288-332.

(13) Tschitschibabin, A. E. Über einige phenylierte Derivate des p, $\mathrm{p}$ - Ditolyls. Berichte der deutschen chemischen Gesellschaft 1907, 40 (2), 1810-1819.

(14) Dong, S.; Li, Z. Recent progress in open-shell organic conjugated materials and their aggregated states. J. Mater. Chem. C 2022, 10, 2431-2449.

(15) Kubo, T.; Shimizu, A.; Sakamoto, M.; Uruichi, M.; Yakushi, K.; Nakano, M.; Shiomi, D.; Sato, K.; Takui, T.; Morita, Y.; et al. Synthesis, intermolecular interaction, and semiconductive behavior of a delocalized singlet biradical hydrocarbon. Angew. Chem. Int. Ed. 2005, 44 (40), 6564-6568. 
(16) Takahashi, T.; Matsuoka, K.-i.; Takimiya, K.; Otsubo, T.; Aso, Y. Extensive Quinoidal Oligothiophenes with Dicyanomethylene Groups at Terminal Positions as Highly Amphoteric Redox Molecules. J. Am. Chem. Soc. 2005, 127 (25), 8928-8929.

(17) Chase, D. T.; Rose, B. D.; McClintock, S. P.; Zakharov, L. N.; Haley, M. M. Indeno[1,2-b]fluorenes: fully conjugated antiaromatic analogues of acenes. Angew. Chem. Int. Ed. 2011, 50 (5), 1127-1130.

(18) Sun, Z.; Huang, K. W.; Wu, J. Soluble and stable heptazethrenebis(dicarboximide) with a singlet open-shell ground state. J. Am. Chem. Soc. 2011, 133 (31), 11896-11899.

(19) Li, Y.; Heng, W.-K.; Lee, B. S.; Aratani, N.; Zafra, J. L.; Bao, N.; Lee, R.; Sung, Y. M.; Sun, Z.; Huang, K.-W.; et al. Kinetically Blocked Stable Heptazethrene and Octazethrene: Closed-Shell or Open-Shell in the Ground State? J. Am. Chem. Soc. 2012, 134 (36), 14913-14922.

(20) Zeng, Z.; Ishida, M.; Zafra, J. L.; Zhu, X.; Sung, Y. M.; Bao, N.; Webster, R. D.; Lee, B. S.; Li, R. W.; Zeng, W.; et al. Pushing extended p-quinodimethanes to the limit: stable tetracyanooligo(N-annulated perylene)quinodimethanes with tunable ground states. J. Am. Chem. Soc. 2013, 135 (16), 6363-6371.

(21) Ni, Y.; Lee, S.; Son, M.; Aratani, N.; Ishida, M.; Samanta, A.; Yamada, H.; Chang, Y. T.; Furuta, H.; Kim, D.; et al. A Diradical Approach towards BODIPY-Based Dyes with Intense NearInfrared Absorption around lambda $=1100 \mathrm{~nm}$. Angew. Chem. Int. Ed. 2016, 55 (8), 2815-2819.

(22) Rudebusch, G. E.; Zafra, J. L.; Jorner, K.; Fukuda, K.; Marshall, J. L.; Arrechea-Marcos, I.; Espejo, G. L.; Ponce Ortiz, R.; Gomez-Garcia, C. J.; Zakharov, L. N.; et al. Diindeno-fusion of an anthracene as a design strategy for stable organic biradicals. Nat. Chem. 2016, 8 (8), 753-759.

(23) Yuen, J. D.; Wang, M.; Fan, J.; Sheberla, D.; Kemei, M.; Banerji, N.; Scarongella, M.; Valouch, S.; Pho, T.; Kumar, R.; et al. Importance of unpaired electrons in organic electronics. $J$. Polym. Sci. Part A: Polym. Chem. 2015, 53 (2), 287-293.

(24) Liu, Y.; Phan, H.; Herng, T. S.; Gopalakrishna, T. Y.; Ding, J.; Wu, J. Toward Benzobis(thiadiazole)-based Diradicaloids. Chem Asian J 2017, 12 (17), 2177-2182.

(25) Tam, T. L. D.; Wu, G.; Chien, S. W.; Lim, S. F. V.; Yang, S.W.; Xu, J. High Spin Pro-Quinoid Benzo[1,2-c;4,5$\mathrm{c}^{\prime}$ ]bisthiadiazole Conjugated Polymers for High-Performance Solution-Processable Polymer Thermoelectrics. ACS Mater. Lett. 2020, 2 (2), 147-152.

(26) Thomas, A.; Bhanuprakash, K.; Prasad, K. M. M. K. Near infrared absorbing benzobis(thiadiazole) derivatives: computational studies point to biradical nature of the ground states. J. Phys. Org. Chem. 2011, 24 (9), 821-832.

(27) Sabuj, M. A.; Huda, M. M.; Sarap, C. S.; Rai, N. Benzobisthiadiazole-based high-spin donor-acceptor conjugated polymers with localized spin distribution. Mater. Adv. 2021, 2 (9), 2943-2955.

(28) Chen, Z.; Li, W.; Sabuj, M. A.; Li, Y.; Zhu, W.; Zeng, M.; Sarap, C. S.; Huda, M. M.; Qiao, X.; Peng, X.; et al. Evolution of the electronic structure in open-shell donor-acceptor organic semiconductors. Nat. Commun. 2021, 12 (1), 5889.

(29) Li, Y.; Li, L.; Wu, Y.; Li, Y. A Review on the Origin of Synthetic Metal Radical: Singlet Open-Shell Radical Ground State? J. Phys. Chem. C 2017, 121 (15), 8579-8588.

(30) Chen, Z.; Li, W.; Zhang, Y.; Wang, Z.; Zhu, W.; Zeng, M.; Li, Y. Aggregation-Induced Radical of Donor-Acceptor Organic Semiconductors. J. Phys. Chem. Lett. 2021, 12 (40), 9783-9790. (31) Lin, Y.; Wang, J.; Zhang, Z. G.; Bai, H.; Li, Y.; Zhu, D.; Zhan, $X$. An electron acceptor challenging fullerenes for efficient polymer solar cells. Adv. Mater. 2015, 27 (7), 1170-1174.

(32) Yuan, J.; Zhang, Y.; Zhou, L.; Zhang, G.; Yip, H.-L.; Lau, T.K.; Lu, X.; Zhu, C.; Peng, H.; Johnson, P. A.; et al. Single-Junction Organic Solar Cell with over 15\% Efficiency Using Fused-Ring
Acceptor with Electron-Deficient Core. Joule 2019, 3 (4), 11401151.

(33) Gao, W.; Lin, F. R.; Jen, A. K. Y. Near - Infrared Absorbing Nonfullerene Acceptors for Organic Solar Cells. Solar RRL 2021, $6(1), 2100868$.

(34) Meng, D.; Zheng, R.; Zhao, Y.; Zhang, E.; Dou, L.; Yang, Y. Near-Infrared Materials: The Turning Point of Organic Photovoltaics. Adv. Mater. 2021, 2107330.

(35) Li, Y.; Li, T.; Lin, Y. Stability: next focus in organic solar cells based on non-fullerene acceptors. Mater. Chem. Front. 2021, 5 (7), 2907-2930.

(36) Li, W.; Liu, D.; Wang, T. Stability Of Non - Fullerene Electron Acceptors and Their Photovoltaic Devices. Adv. Funct. Mater. 2021, 2104552.

(37) Che, Y.; Niazi, M. R.; Izquierdo, R.; Perepichka, D. F. Mechanism of the Photodegradation of A-D-A Acceptors for Organic Photovoltaics. Angew. Chem. Int. Ed. 2021, 60, 2483324837.

(38) Liu, H.; Wang, W.; Zhou, Y.; Li, Z. a. A ring-locking strategy to enhance the chemical and photochemical stability of A-D-Atype non-fullerene acceptors. J. Mater. Chem. A 2021, 9 (2), 10801088.

(39) Cui, Y.; Liu, J.; Li, Z.; Ji, M.; Zhao, M.; Shen, M.; Han, X.; Jia, T.; Li, C.; Wang, Y. Donor - Acceptor - Type Organic Small - Molecule - Based Solar - Energy - Absorbing Material for Highly Efficient Water Evaporation and Thermoelectric Power Generation. Adv. Funct. Mater. 2021, 31 (49), 2106247.

(40) Kim, Y.; Kim, Y. J.; Kim, Y. A.; Jung, E.; Mok, Y.; Kim, K.; Hwang, H.; Park, J. J.; Kim, M. G.; Mathur, S.; et al. Open-Shell and Closed-Shell Quinoid-Aromatic Conjugated Polymers: Unusual Spin Magnetic and High Charge Transport Properties. ACS Appl. Mater. Interfaces 2021, 13 (2), 2887-2898.

(41) Wang, Y.; Liu, J.; Cui, Y.; Pan, Y.; Chen, Z.; Jia, T.; Li, C. Donor-Acceptor Molecule Based High Performance Photothermal Organic Material for Efficient Water-Electric Cogeneration. Angew. Chem. Int. Ed. 2022, 202117087.

(42) Li, X.; Fang, F.; Sun, B.; Yin, C.; Tan, J.; Wan, Y.; Zhang, J.; Sun, P.; Fan, Q.; Wang, P.; et al. Near-infrared small molecule coupled with rigidness and flexibility for high-performance multimodal imaging-guided photodynamic and photothermal synergistic therapy. Nanoscale Horiz. 2021, 6 (2), 177-185.

(43) Zhang, H.; Yao, H.; Hou, J.; Zhu, J.; Zhang, J.; Li, W.; Yu, R.; Gao, B.; Zhang, S.; Hou, J. Over 14\% Efficiency in Organic Solar Cells Enabled by Chlorinated Nonfullerene Small-Molecule Acceptors. Adv. Mater. 2018, 30 (28), e1800613.

(44) Zhang, Y.; Yao, H.; Zhang, S.; Qin, Y.; Zhang, J.; Yang, L.; Li, W.; Wei, Z.; Gao, F.; Hou, J. Fluorination vs. chlorination: a case study on high performance organic photovoltaic materials. Sci China Chem 2018, 61 (10), 1328-1337.

(45) Li, S.; Zhan, L.; Zhao, W.; Zhang, S.; Ali, B.; Fu, Z.; Lau, T.K.; Lu, X.; Shi, M.; Li, C.-Z.; et al. Revealing the effects of molecular packing on the performances of polymer solar cells based on A-D-C-D-A type non-fullerene acceptors. J. Mater. Chem. A 2018, 6 (25), 12132-12141.

(46) Chen, T.-W.; Peng, K.-L.; Lin, Y.-W.; Su, Y.-J.; Ma, K.-J.; Hong, L.; Chang, C.-C.; Hou, J.; Hsu, C.-S. A chlorinated nonacyclic carbazole-based acceptor affords over $15 \%$ efficiency in organic solar cells. J. Mater. Chem. A 2020, 8 (3), 1131-1137.

(47) Cao, C.; Lai, H.; Chen, H.; Zhu, Y.; Pu, M.; Zheng, N.; He, F. Over $17.5 \%$ Efficiency Ternary Organic Solar Cells with Enhanced Photon Utilization via a Medium Band Gap Non-Fullerene Acceptor. J. Mater. Chem. A 2021, 9, 16418.

(48) Sun, Z.; Ye, Q.; Chi, C.; Wu, J. Low band gap polycyclic hydrocarbons: from closed-shell near infrared dyes and semiconductors to open-shell radicals. Chem. Soc. Rev. 2012, 41 (23), 7857-7889. 
(49) Wang, Y.; Zhu, W.; Du, W.; Liu, X.; Zhang, X.; Dong, H.; Hu, W. Cocrystals Strategy towards Materials for Near-Infrared Photothermal Conversion and Imaging. Angew. Chem. Int. Ed. 2018, 57 (15), 3963-3967.

(50) Lu, B.; Chen, Y.; Li, P.; Wang, B.; Mullen, K.; Yin, M. Stable radical anions generated from a porous perylenediimide metalorganic framework for boosting near-infrared photothermal conversion. Nat. Commun. 2019, 10 (1), 767.

(51) Wang, D.; Kan, X.; Wu, C.; Gong, Y.; Guo, G.; Liang, T.; Wang, L.; Li, Z.; Zhao, Y. Charge transfer co-crystals based on donor-acceptor interactions for near-infrared photothermal conversion. Chem. Commun. 2020, 56 (39), 5223-5226.

(52) Chen, Z.; Su, Y.; Tang, X.; Zhang, X.; Duan, C.; Huang, F.; Li, Y. Manipulating Grain Boundary Defects in $\pi$ - Conjugated Covalent Organic Frameworks Enabling Intrinsic Radical Generation for Photothermal Conversion. Sol. RRL 2021, 5 (12), 2100762.

(53) Chu, Y.; Liao, S.; Liao, H.; Lu, Y.; Geng, X.; Wu, D.; Pei, J.; Wang, Y. Second Near-Infrared Photothermal Therapy with Superior Penetrability through Skin Tissues. CCS Chemistry 2021, 3289-3300.

(54) Zhang, X.; Li, Y.; Chen, Z.; Li, P.; Chen, R.; Peng, X. Molecular engineering of narrow bandgap porphyrin derivatives for highly efficient photothermal conversion. Dyes Pigm. 2021, $192,109460$.

(55) Zhang, Y.; Wu, G.; Liu, H.; Tian, R.; Li, Y.; Wang, D.; Chen, R.; Zhao, J.; Liu, S.; Li, Z.; et al. Donor-acceptor based twodimensional covalent organic frameworks for near-infrared photothermal conversion. Mater. Chem. Front. 2021, 5 (17), 65756581.

\section{Table of Contents}

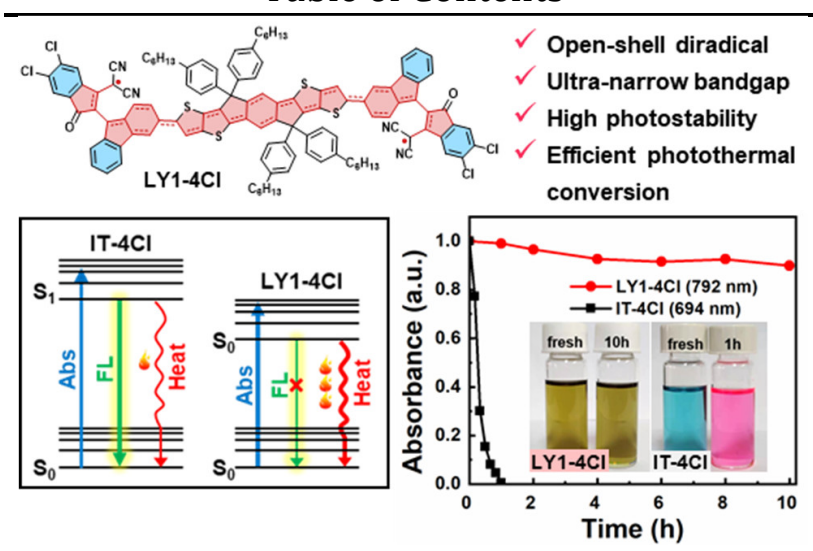

